\title{
Minimal order disturbance estimator design for aircraft load alleviation control
}

\author{
Daniel Ossmann ${ }^{1}$ and Charles Poussot-Vassal ${ }^{2}$
}

\begin{abstract}
In this paper a novel approach to design linear disturbance estimators is presented. The model based design approach relies on advanced nullspace computation techniques and explicit facilitates an estimators design of low state dimension. This reduces the dependencies on the underlying design models and thereby increases robustness against modeling errors. To further support this low order characteristics an advanced model approximation technique for preprocessing high order design models is presented. The proposed approaches are applied to a generic model of a business jet aircraft. The derived disturbance estimator is used in a control algorithm to reduce the wing bending moments on the aircraft in case of wind gusts. Both, the disturbance estimator and the load alleviation controller are verified in a non-linear closed loop aircraft simulation model.
\end{abstract}

\section{INTRODUCTION}

Estimating an unknown disturbance using linear observers and estimators can be powerful in conjunction with feedback control [1]. The idea is to appropriately include a disturbance estimate in the control law so that disturbance effects can be approximately removed. This requires the estimation of the disturbance with high accuracy. There is a long history on disturbance estimator design and thus a rich literature available on different design methods. The simplest design idea arises in the frequency domain, where the known, disturbance free plant model is used to derive the difference between the measured and estimated output, which is considered to be the disturbance. See for example references [2], [3] for description and proper applications. The unknown input observer [4], [5], [6] translates the problem into the time and state-space domain and estimates both the systems states and the disturbance. This requires the definition of disturbance dynamics to allow the disturbance estimation via a dedicated system state. The problem formulation in the time domain and the introduction of disturbance states also opened the door to the adaption of well developed state estimators as Kalman filters or $\mathcal{H}_{\infty}$-norm based filter designs for the disturbance estimation problem [7]. The similarity of the Kalman filter and the classical designs in the frequency domain are discussed in [8].

The topic of disturbance estimators is closely related to the approaches used in the fault detection and isolation (FDI) community. Interpreting the appearing faults as disturbances, a lot of approaches available in the FDI literature can be used

\footnotetext{
${ }^{1}$ Dr. Daniel Ossmann is with the Institute of System Dynamics and Control, German Aerospace Center, Muenchener Strasse 20, 82234 Wessling, Germany Daniel.Ossmann@dlr.de

${ }^{2}$ Dr. Charles Poussot-Vassal is with the Systems and Signal Processing Department, ONERA Centre de Toulouse, 2 Avenue Edouard Belin, 31000 Toulouse, France Charles.Poussot-Vassal@onera.fr
}

for the estimation of disturbances. The main difference between FDI and disturbance estimation is, that the disturbance observer has to accurately reflect the disturbance input signal, while FDI aims to generate any signal, which is decoupled from all known and unknown inputs but the fault(s) to be detected. However, some approaches can be adapted to not only provide fault detection but also fault estimation, i.e., reflecting the actual value of the fault. This fault estimation problem is comparable to the disturbance estimation problem and methods used for the one can be used for the other. Notable linear design methods to solve FDI problems are Luenberger observers [9] and Luenberger-type approaches using for example geometric methods [10] or eigenstructure assignment [11]. Further, also arising from the idea of disturbance observers, unknown input observers [11] are well established in the field of FDI. Beside the observer based approaches, parity space methods [12], [13] and nullspace based approaches have been introduced, see e.g., [14], [15]. These two methods allow the design of detectors of minimal order. The minimal order characteristics of the observer reduce the dependence on the underlying model dynamics and thereby increases implicitly the robustness to modeling errors and model uncertainties. The main difference in the two mentioned approaches lies in the computational and numerical manner in solving the decoupling conditions. The nullspace based design methods can be considered to be numerically more reliable [16].

In this paper we make use of the latest developments in the nullspace based design methods in the FDI community to present a novel approach for the design of disturbance estimators. Originally developed to solve FDI problems [14], [17], herein the disturbance estimation problem is stated in a form, which allows its direct solving via the idea of nullspace computations. The design methodology to design a minimal order disturbance estimator is introduced in section II. In case of design models with very high state dimension, which are for example usually present when dealing with load alleviation problems on aircraft, an a priori model reduction is recommended. Thus, in section III an advanced model approximation technique is additionally discussed to facilitate the minimal order estimator design. A main focus of the paper lies on the realistic and industrially relevant application. In section IV a generic example of a medium size business jet is introduced. It serves as basis for the order reduction and the subsequent disturbance observer design. The model is further utilized in section $\mathrm{V}$ to verify the load alleviation capabilities of the proposed load alleviation system. The idea of this load alleviation system is to use 
the wind estimate to trigger a dedicated feedback law, which alleviates the maximum bending moments on the wings in case of increased wind gusts. A description of this non-linear control law is provided in section IV.C.

\section{Disturbance ESTIMATOR DESIGN}

In this section the disturbance estimator design problem is generally derived. It is shown how it is connected to the idea of nullspace computations for linear dynamical systems.

\section{A. Problem formulation}

Consider the linear model described by the input-output form

$$
y(s)=G_{u}(s) u(s)+G_{\bar{d}} \bar{d}(s)+G_{d} d(s),
$$

where $y(s), u(s)$, and $\bar{d}(s)$ are the Laplace-transformed vectors of the $p$-dimensional system output vector $y(t)$, the $m_{u}$-dimensional control input vector $u(t)$, and the $m_{\bar{d}^{-}}$ dimensional disturbance vector $\bar{d}(t)$, respectively. $d(s)$ is the Laplace-transformed of scalar disturbance input $d(t)$ to be estimated. $G_{u}(s), G_{\bar{d}}(s)$, and $G_{d}(s)$ are the transfer-function matrices (TFMs) from control inputs to outputs, disturbance inputs to outputs, and disturbance input to be estimated to outputs, respectively. Note that only one disturbance $d(s)$ out of the full disturbance vector $[\bar{d}(s) d(s)]^{T}$ shall be estimated. Thus, a disturbance estimate $\tilde{d}(s)$ needs to be decoupled from any additional disturbances $\bar{d}(s)$ acting on the system. This is no restriction to the design process as for $N_{d}=m_{\bar{d}}+m_{d}$ disturbances acting on the system, $N_{d}$ individual estimators can be designed using the methods described below, if the according design conditions are fulfilled.

For the system described in (1) a linear disturbance estimator processes the measurable system outputs $y(t)$ and control inputs $u(t)$ and generates the disturbance estimate $\tilde{d}(t)$. In the input-output form this can be described by

$$
\tilde{d}(s)=O_{e}(s)\left[\begin{array}{l}
y(s) \\
u(s)
\end{array}\right]
$$

where $O_{e}(s)$ is the TFM of the disturbance estimator. For a physically realizable disturbance estimator, $O_{e}(s)$ must be proper and stable. The order of $O_{e}(s)$ is the dimension of the state vector of a minimal state-space realization of $O_{e}(s)$. The general description in (2) allows the definition of the disturbance estimation problem (DEP):

Design a physically realizable linear disturbance estimator of the form (2) such that for all $u(t)$ and $\bar{d}(t)$

$$
\begin{array}{ll}
\text { (i) } & \tilde{d}(t)=0 \text { when } d(t)=0 \\
\text { (ii) } & \tilde{d}(t) \approx d(t) \text { when } d(t) \neq 0 \\
\text { (iii) } & \tilde{d}(t) \text { is asymptotically bounded. }
\end{array}
$$

Additionally, $O_{e}(s)$ shall be of minimal order $(i v)$.

\section{B. Solving the disturbance estimation problem}

The requirements $(i)$ and $(i i)$ of the DEP are easily transformed into algebraic conditions. Therefore, the system's input-output equation (1) is inserted in the disturbance estimator equation (2), so that the disturbance estimate depends on the control and disturbance inputs, i.e.,

$$
\tilde{d}(s)=O_{e}(s)\left[\begin{array}{ccc}
G_{u}(s) & G_{\bar{d}}(s) & G_{d}(s) \\
I_{m_{u}} & 0 & 0
\end{array}\right]\left[\begin{array}{c}
u(s) \\
\bar{d}(s) \\
d(s)
\end{array}\right] .
$$

The decoupling condition $(i)$ requires that the disturbance estimate $\tilde{d}(s)$ is decoupled from all inputs $u(s)$ and disturbances $\bar{d}(s)$, which shall not be estimated. This is equivalent to demand

$$
O_{e}(s)\left[\begin{array}{cc}
G_{u}(s) & G_{\bar{d}}(s) \\
I_{m_{u}} & 0
\end{array}\right]:=O_{e}(s) G_{n}(s)=0 .
$$

It follows that $O_{e}(s)$ needs to be a left annihilator of $G_{n}(s)$. By deriving a minimal basis $N_{l}(s)$ for the left nullspace of $G_{n}(s)$, the design conditions $(i)$ can be tackled. For the design condition $(i i)$, the basic constraint

$$
O_{e}(s)\left[\begin{array}{c}
G_{d}(s) \\
0
\end{array}\right] \neq 0
$$

must be fulfilled. This can only be achieved, if the DEP is actually solvable. The solve-ability condition follows the theorem presented in [16]:

Theorem 1: For the system (1) the DEP is solvable if and only if

$$
\operatorname{rank}\left(\left[\begin{array}{ll}
G_{\bar{d}}(s) & G_{d}(s)
\end{array}\right]\right)>\operatorname{rank}\left(G_{\bar{d}}(s)\right) .
$$

Thus, the disturbance to be estimated needs to be linearly independent of all the other disturbances. Note that the condition (6) is independent of the control input $u$. Thus, for the case $m_{\bar{d}}=0$, the DEP is always solvable. This makes sense as just known inputs and one unknown input to be estimated act on the system.

If condition (6) is fulfilled, a disturbance observer fulfilling (ii) which is stable (iii) as well as proper and minimal (iv) can be constructed by linear combination of the rows of the left nullspace basis $N_{l}(s)$ of $G_{n}(s)$ [16], i.e.,

$$
O_{e}(s)=h(s) N_{l}(s) \text {. }
$$

Note that any linear combination of $N_{l}(s)$ solves the design condition $(i)$ but it has to be checked if (5) is ensured to fulfill condition $(\mathrm{ii})$. To solve the DEP, the transfer function vector is defined as $h(s)=k w(s)$, which consists of a transfer function vector $w(s)$ to make the filter proper, stable and of least order. The constant $k$ is used for scaling to ensure a transfer behavior from $d$ to $\tilde{d}$ of 1 . If the DEP is solvable, it is always possible to freely select the scaling $k$. As $k$ is selected to be a constant scalar herein, its value is chosen based on the transfer gain from the disturbance input to the derived disturbance estimator output at a certain frequency. Note that this does usually not restrict the tracking of the disturbance to a single frequency, as disturbances often show constant gain over a wider frequency range. More details on the actual computation are presented next. 


\section{Computation}

The expression (7) represents a parametrization of all possible disturbance estimators and is the basis of the socalled nullspace based filter design methods. These methods have been formally introduced in [14] using polynomial bases and have been extended to be numerically more reliable algorithms in [15], [16] using rational basis, both for fault detection purposes. Successful applications of the approaches can be found in [18], [19], [20].

The slightly modified computation procedure to compute a disturbance estimator using these methods herein can be summarized in three dedicated steps. If condition (6) is fulfilled,

(I.) compute a minimal left nullspace basis $N_{l}(s)$ of $G_{n}(s)$,

(II.) compute a rational vector $w(s)$ such that $\tilde{O}_{e}(s)=w(s) N_{l}(s)$ is proper, stable, has least order, and (5) is fulfilled,

(III.) select a constant $k$ s.t. $O_{e}(s)=k W(s) N_{l}(s)$ ensures a transfer from $d$ to $\tilde{d}$ of 1 at a defined frequency.

For steps $(I$.$) and (I I$.$) we rely on the numerically sound$ approach presented in [21]. It allows to derive a rational nullspace basis allowing to generate a minimal, proper and stable disturbance observer $\tilde{O}_{e}(s)$. In more detail, step $(I$.) is solved using matrix pencil methods. The minimal order in $(I I$.$) is then ensured via solving a minimum dynamic cover$ problem and the stable and proper dynamics are achieved via a QR decomposition. Interested readers in the computational ideas and their details are referred to [17].

One essential difference to the fault detection problem is the requirement that in case of a disturbance estimator the estimator's output shall reflect the disturbance magnitude. This is required as this estimate is usually directly used as input signal in a feedback controller. Thus, to allow the fault estimation the correct scaling of the observer (III.) needs to be explicitly considered. If the DEP is solvable, condition (4) holds for any observer $\tilde{O}_{e}(s)$ derived via (7). Thus, equation (3) reduces to

$$
\tilde{d}(s)=\left[\begin{array}{ll}
\tilde{O}_{y}(s) & \tilde{O}_{u}(s)
\end{array}\right]\left[\begin{array}{l}
y(s) \\
u(s)
\end{array}\right]=\tilde{O}_{y}(s) G_{d}(s) d(s),
$$

where $\tilde{O}_{e}(s)=\left[\tilde{O}_{y}(s) \tilde{O}_{e, u}(s)\right]$ is separated into $\tilde{O}_{e, y}(s)$, which processes the system measurements $y$, and into $\tilde{O}_{u}(s)$, which processes the (known) control inputs $u$. Note that $\tilde{O}_{e, y}(s) G_{d}(s)$ is one single-input single-output transfer function as only scalar disturbances $d$ are considered in (1). Thus, considering the requirement that the magnitude of $\tilde{d}$ follows $d$ and moving into the frequency domain, leads to

$$
k\left|\tilde{O}_{e, y}(\imath \omega) G_{d}(\imath \omega)\right|=1 .
$$

To follow the disturbance signal $d$ at a desired frequency $\omega_{d}$, the scaling for the disturbance observer $O_{e}(s)$ can be selected as $k=\left|\tilde{O}_{e, y}\left(\imath \omega_{d}\right) G_{d}\left(\imath \omega_{d}\right)\right|^{-1}$. As an example, if the steady state value of the disturbance estimate shall equal the steady state value of the disturbance, $\omega_{d}=0$ is an appropriate choice. As the dynamics of the disturbance observer can be freely chosen [17], a small lag between the disturbance and its estimate is ensured via setting adequately fast disturbance observer dynamics.

In case of linear design models of very high state dimensions, which are, e.g., present when dealing with structural dynamics of aircraft, an a priori approximation of the high order model is recommended. The theoretical background used for the approximation of the aircraft models in this paper is provided next.

\section{MODEL APPROXIMATION}

When dealing with industrial problems such as aircraft systems, associated models usually embed unsteady aerodynamics as well as structural modes and aerodynamic delays. Consequently, the dimension of the state-space dimensions can be very large, and additionally models can include delays and potentially mixing differential and algebraic equations. Thus, before the methods presented in section II can be applied, a pre-processing step, to reduce the state dimension and simplify the complexity should be first applied in order to improve the numerical treatment and accuracy of the results. A short reminder of the methods involved in section IV are briefly discussed in this section. As these methods are not the main topic of this paper, more details on infinite or datadriven model approximation can be found in [22], [23], [24] and on finite order large-scale model approximation in [25], [26]. Let us follow these two classes of problems and remind the driving ideas as follows.

\section{A. Infinite dimensional or data-driven model approximation}

Given an infinite dimensional model $H$, which results, e.g., if time-delays are considered, it is possible to obtain the frequency-domain responses $\Phi_{i} \in \mathbb{C}^{n_{y} \times n_{u}}$ for different frequency samples $\omega_{i}(i=1, \ldots, N)$. Then, one can write $H\left(\imath \omega_{i}\right)=\Phi_{i}$. One of the data-driven approaches is based on the interpolation framework well defined in [27], [22], involving the Loewner matrices. The method consists of an exact rational model interpolation, optionally followed by a reduction procedure. To this aim, let us first partition the collected data $\left(\omega_{i}, \Phi_{i}\right)_{i=1}^{N}$ in two disjoint sets as follows $(N=q+k)$ :

$$
\begin{aligned}
\imath\left[\omega_{1}, \ldots, \omega_{N}\right] & =\left[\mu_{1}, \ldots, \mu_{q}\right] \cup\left[\lambda_{1}, \ldots, \lambda_{k}\right] \\
{\left[\Phi_{1}, \ldots, \Phi_{N}\right] } & =\left[\tilde{v}_{1}, \ldots, \tilde{v}_{q}\right] \cup\left[\tilde{w}_{1}, \ldots, \tilde{w}_{k}\right] .
\end{aligned}
$$

Then, define $l_{j} \in \mathbb{C}^{n_{y} \times 1}(j=1, \ldots, q)$ and $r_{i} \in \mathbb{C}^{n_{u} \times 1}$ $(i=1, \ldots, k)$ the $q$ left and $k$ right tangential directions. Using these tangential directions, let us define $v_{j}^{*}=$ $l_{j}^{*} \tilde{v}_{j} \in \mathbb{C}^{1 \times n_{u}}$ and $w_{i}=\tilde{w}_{i} r_{i} \in \mathbb{C}^{n_{y} \times 1}$ the left and right tangential data directions, respectively. Based on the left interpolation driving frequencies $\left\{\mu_{i}\right\}_{i=1}^{q} \in \mathbb{C}$ with left output or tangential directions $\left\{l_{i}\right\}_{i=1}^{q} \in \mathbb{C}^{n_{y}}$, producing the left responses $\left\{v_{i}\right\}_{i=1}^{q}$ and right interpolation driving frequencies $\left\{\lambda_{i}\right\}_{i=1}^{k} \in \mathbb{C}$ with right input or tangential directions $\left\{r_{i}\right\}_{i=1}^{k} \in \mathbb{C}^{n_{u}}$, producing the right responses $\left\{w_{i}\right\}_{i=1}^{k}$, the objective is to find a model transfer function $\tilde{H}$ 
which is a tangential interpolation of the data, i.e., satisfies the following left and right interpolation conditions:

$$
\left.\begin{array}{c}
l_{j}^{*} \tilde{H}\left(\mu_{j}\right)=v_{j}^{*} \\
\text { for } j=1, \ldots, q
\end{array} \text { and } \begin{array}{c}
\tilde{H}\left(\lambda_{i}\right) r_{i}=w_{i} \\
\text { for } i=1, \ldots, k
\end{array}\right\} .
$$

The interpolation problem (11) can be solved thanks to the Loewner framework (see, e.g., [27]). An important property of the Loewner approach is that it encodes the minimal McMillian degree of the interpolation model and its minimal realization order $n$. This then leads to an exact descriptor model interpolating the data, especially useful, when the number of data is very large.

\section{B. Finite dimensional model approximation}

Once an exact interpolation model $\tilde{H}$ has been obtained, with potentially large dimension, a second step then consists in approximating this finite order model with a low dimensional one. One common objective in model approximation consists in finding a reduced-order model that well captures the main original input/output dynamical behavior. To address this objective, the (frequency-limited) $\mathcal{H}_{2}$-norm mismatch error is commonly used, see e.g., [26], [28]. The resulting approximation problem consists thus in seeking a low order approximation model $\hat{H}(s)$ of $\tilde{H}(s)$, such that:

$$
\hat{H}:=\arg \min _{\substack{G \in \mathcal{H}_{2}^{n_{y} \times n_{u}} \\ \operatorname{rank}(G) \stackrel{=}{=} r \ll n}}\|\tilde{H}-G\|_{\mathcal{H}_{2} .} .
$$

Beside the fact that problem (12) is non convex and nonlinear, some conditions have been proposed to reach the socalled first order optimality conditions and procedures to ensure that a local (hopefully global) optimum is reached. Moreover, the proposed IRKA and FL-ISTIA algorithms are appropriate to practically tackle these problems (see e.g., [26], [28] for details). Finally, the derived model $\hat{H}$ can be easily brought into the form (1) by separating the inputs accordingly.

\section{Application}

Reducing structural loads on an aircraft by advanced control techniques is a main research interest of today's aircraft industry. Reducing the loads allows building a lighter aircraft and thereby saves costs. The loads arise from steering the aircraft (maneuver loads) and from external disturbance inputs, (gust loads). The latter are considered in this paper for a generic business jet aircraft.

For being able to realistically compute and compare loads with and without load alleviation controller, while taking into account as much as possible structural interactions, an aircraft model with about 300 states is available. The model includes detailed structural and aerodynamic effects, delays, realistic actuator and sensor models as well as a baseline control law, providing adequate handling qualities to the pilot. The control inputs to the open loop aircraft model are the commands of the elevator, the inner ailerons and the outer ailerons. The gust impact on the model is characterized by nine inputs describing the position, velocity and acceleration impact at three different locations along the fuselage. As measured signals for feedback control, the pitch rate, the load factor and the angle of attack are provided. The provided baseline controller only commands the elevator, while the load alleviation controller shall use the ailerons symmetrically to alleviate the bending moments due to gusts.

The open loop model, i.e., without baseline controller, serves as basis for the disturbance observer design. The design will be carried out for a fixed aircraft speed and altitude as an example and verified in the full non-linear, closed loop aircraft model including a case with varying mass and center of gravity position.

\section{A. Model approximation}

The high number of states as well as the nine disturbance inputs makes the estimator and control design challenging. Thus the idea is to reduce the state dimension and the number of disturbance inputs. In view of disturbance estimator and control design, it is preferable to use a single disturbance single input.

The nine disturbance inputs are actually used to model a single gust hitting three different parts (front, middle, rear) of the aircraft one after another. The derivatives of the three gust input positions are required to consider the unsteady aerodynamics. Consequently, the second and third set of gust inputs are equal to the first one but delayed by a fixed time delay. Thus, mathematically the second and third set of inputs can be derived by simply delaying the first on. Additionally, the velocity and acceleration of the first gust input can be derived by derivative action on the first gust input position, finally reducing the gust inputs to a single one. After these modifications, the resulting model now embeds two internal delays (denoted $\tau_{1}$ and $\tau_{2}$, related to the velocity of the aircraft) and has a rank deflective descriptor form. These internal delays are explained by the use of exact delay actions applied on the exact derivative terms. Thus, the linear timeinvariant dynamical system can be represented by a first order descriptor realization with $n_{u}$ inputs (including one single gust input), $n_{y}$ outputs, $n_{x}$ internal variables, and the two internal delays. The model is given by a set of differential and algebraic equations

$$
\begin{aligned}
E \dot{x}(t) & =A_{0} x(t)+A_{1} x\left(t-\tau_{1}\right)+A_{2} x\left(t-\tau_{2}\right)+B u(t) \\
y(t) & =C x(t),
\end{aligned}
$$

with the rank defective matrix $E$, the internal variables $x(t) \in \mathbb{R}^{n_{x}}:=\mathcal{X}$, and the the input and output functions $u(t) \in \mathbb{R}^{n_{u}}:=\mathcal{U}$ and $y(t) \in \mathbb{R}^{n_{y}}:=\mathcal{Y}$, respectively. $E, A_{0}, A_{1}, A_{2} \in \mathbb{R}^{n_{x} \times n_{x}}, B \in \mathbb{R}^{n_{x} \times n_{u}}$ and $C \in \mathbb{R}^{n_{y} \times n_{x}}$ are constant matrices. The matrix pencil is regular if the matrix $\left(A_{0}+A_{1} e^{-\tau_{1} \lambda}+A_{2} e^{-\tau_{1} \lambda}\right)-\lambda E$ is non-singular for some finite $\lambda \in \mathbb{C}$ and a $\left\{\tau_{1}, \tau_{2}\right\}$ couple. In this case, the associated transfer function is

$$
H(s)=C\left(s E-A_{0}-A_{1} e^{-\tau_{1} s}-A_{2} e^{-\tau_{2} s}\right)^{-1} B+D .
$$

Obviously, due to the presence of delays in the dynamical part of the equations, the resulting model is now of infinite dimension. To cope with this, first an exact stable Loewner 
interpolation of this infinite model by a finite order one is performed as discussed in section III-A. This leads to a large scale descriptor model of dimension $n$, which exactly interpolates the infinite dimensional model $H$, with $\tilde{H}(s)$, of the form

$$
\tilde{H}(s)=\tilde{C}(s \tilde{E}-\tilde{A})^{-1} \tilde{B} .
$$

This finite order model (e.g., with a finite number of eigenvalues) can now be approximated using any (frequencylimited) $\mathcal{H}_{2}$ oriented model approximation techniques as discussed in section III-B and further presented in e.g., [26], [29], [30]. This leads to a reduced order model with dimension $r \ll n$, as,

$$
\hat{H}(s)=\hat{C}(s \hat{E}-\hat{A})^{-1} \hat{B}
$$

which minimizes the (frequency-limited) mismatch error. Finally, the aircraft model of about 300 states with nine disturbance inputs has been approximated with a model of order $r=25$ with a single disturbance input. This reduced model can now be used to design the disturbance estimator.

\section{B. Estimator Design}

A disturbance estimator with the presented methods in section II is designed for the aircraft. The final design model after the model approximation features 25 states, three control inputs, namely elevator, symmetric inner aileron and symmetric outer aileron positions, one gust input and the two measurable outputs, i.e., pitch rate and the load factor. Applying the approach presented in section II, results in a first order disturbance estimator. As the dynamics of the detector can be freely chosen, the single real pole is set to -1 . This value still provides an adequate estimation delay and ensures reasonable estimator gains. The resulting state-space representation of the disturbance estimator is given by

$$
\begin{aligned}
\dot{x}_{e} & =-x_{e}+B_{e}\left[\begin{array}{lllll}
q & n_{z} & \eta & \xi_{i} & \xi_{o}
\end{array}\right]^{T} \\
\tilde{d} & =x_{e}+D_{e}\left[\begin{array}{lllll}
q & n_{z} & \eta & \xi_{i} & \xi_{o}
\end{array}\right]^{T},
\end{aligned}
$$

where $x_{e}$ is the scalar detector state, $q$ the pitch rate, $n_{z}$ the load factor, $\eta$ the commanded elevator position, $\xi_{i}$ the commanded symmetric inner aileron position, and $\xi_{o}$ the commanded symmetric outer aileron position. $B_{e}$ and $D_{e}$ are the $1 \times 5$ input and feed-through vectors of the observer, respectively. The disturbance acts on the plant output with differentiating behavior at low frequencies, i.e.., constant gusts are not visible in the measurements. As the gusts to be estimated are dynamic 1-cosine gusts, the frequency to determine the scaling factor $k$ is selected at $w_{d}=50 \mathrm{rad} / \mathrm{s}$ to ensure an adequate tracking of the incoming gusts.

In Fig. 1 the response of the developed first order disturbance observer to vertical 1-cosine gust inputs is presented for two different mass and center of gravity cases. The first case is referred to as design case in the following, as at this mass and center of gravity position the reduced order design model has been derived. The second case is referred to as verification case as it is used to verify the robustness of the observer against changes in mass and center of gravity positions. The shown simulation results are generated using the full non-linear, closed-loop simulation model. Two different gust wavelengths of $33 \mathrm{~m} / \mathrm{s}$ (first diagram) and $84 \mathrm{~m} / \mathrm{s}$ (second diagram) corresponding to a vertical gust velocity of around $13.5 \mathrm{~m} / \mathrm{s}$ and $16 \mathrm{~m} / \mathrm{s}$ are depicted. In both cases the input gust is estimated adequately. In all four cases the estimations show a slight delay compared to the actual gust. This is due to the fact that the disturbance acts on the front part of the aircraft first. The sensed pitching moments however, result from the gust acting on the wing, i.e., the middle part of the aircraft. Thus, the resulting delay in the estimation is approximately the time the gust needs to travel from the front of the aircraft to the wing section.

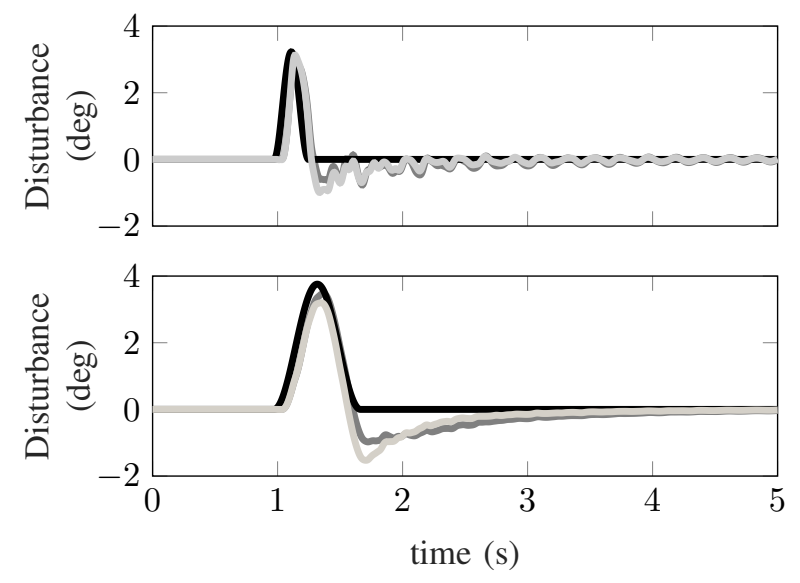

Fig. 1. Estimated disturbances for the design ( - ) and verification $(-)$ case compared to the real disturbances $(-$ ) for a gust wavelength of $33 \mathrm{~m} / \mathrm{s}$ (first diagram) and $84 \mathrm{~m} / \mathrm{s}$ (second diagram).

Besides the actual estimation performance, the decoupling of the control inputs needs to be ensured. To test the decoupling, a step-like elevator input of 10 degree over 5 seconds is used to simulate a pilot input command. In Fig. 2 the resulting estimator outputs for the design and verification case are depicted. As expected, the estimate is not equal zero due to the consideration of the full aircraft model. However, it is sufficiently small, so that a disturbance can be distinguished from these unwanted signal excitations by setting an adequate threshold. Note that for the design case ( - ) the signal is naturally smaller compared to the verification case $(-)$.

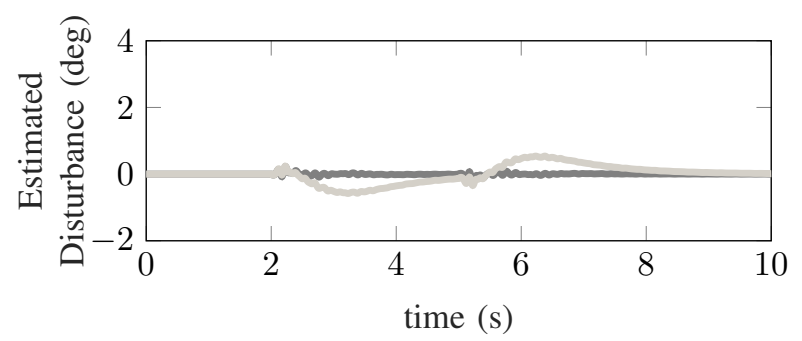

Fig. 2. Disturbance estimator response to a step-like elevator input for the design ( - ) and the verification $(-$ ) case to verify the control input decoupling. 


\section{Control Strategy}

Having an estimate of the gust available, a non-linear control strategy is developed. When a gust hits the aircraft, the increased angle of attack on the wings leads to an increased lift on the wings. This lift increases the encountered wing bending moment. The idea is to symmetrically and rapidly deflect the ailerons, so that the additional lift resulting from the wind gust gets reduced. If the disturbance estimate exceeds a defined threshold value, the ailerons are deflected, where the direction of the deflection depends on the gust direction. This direction is determined from the change in the (sensed) angle of attack $\alpha$. The proposed control law is a bang-bang like controller with a threshold based triggering. The threshold is selected based on simulations. The controller stays active as long the gust estimate exceeds its threshold but at least one second after activation to avoid a rapid on and off switching.

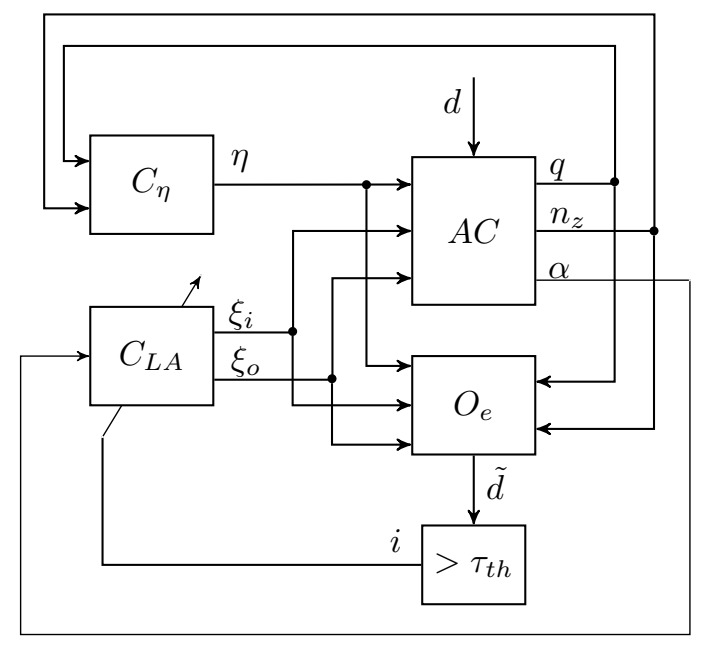

Fig. 3. Closed loop structure including aircraft dynamics, baseline controller, disturbance observer, triggering logic, and load alleviation controller.

The overall structure of the closed loop system is depicted in Fig. 3. The baseline controller $C_{\eta}$ uses the measurements load factor $n_{z}$ and the pitch rate $q$ from the aircraft $A C$ as inputs to generate the elevator deflection $\eta$. It ensures adequate handling and disturbance rejection in the longitudinal, rigid body motion of the aircraft. The disturbances $d$ are acting on the aircraft as unknown inputs. The aircraft in this illustration also includes the sensor and actuator dynamics. If the gust estimate $\tilde{d}$ of the estimator $O_{e}$ is above the defined threshold $\tau_{\text {th }}$, the load alleviation is triggered via the Boolean variable $i$. The angle of attack $\alpha$ is used to determine the direction of the load alleviation aileron command.

\section{Closed LOOP LOAD VERIFICATION}

This section reports the verification results gathered from the full order closed loop model of the aircraft. The closed loop includes besides the full aircraft dynamics, the baseline controller, the load reduction control strategy, second order actuator models, and second order sensor dynamics with time delays. For the load alleviation quantification a full spectrum of 1-cosine gusts acting vertically from below and above are simulated. Gust wavelengths from $9 \mathrm{~m} / \mathrm{s}$ to $107 \mathrm{~m} / \mathrm{s}$ are simulated, resulting in 22 gust simulations. During each gust simulation, the maximum value of the bending moment on six different wing-span locations is recorded and the worst case over the 22 simulations is calculated at each span wing location. The wing span of the aircraft is around 11.9 meters. The results are then normalized with the corresponding maximum allowable value of the bending moment at the specific locations. For comparison the simulations are performed once with and once without gust load alleviation controller activated.

In Fig. 4 the results of the described load verification are presented. For both, the design case and the verification case, the loads are decreased. While for the baseline controller the worst case over the wing is around 1, i.e., matching the maximum allowable loads, the worst case when including the load alleviation controller is around 0.94 at a wing span of around $10 \mathrm{~m}$, achieving a worst case load reduction of about $6 \%$. As load reduction results in both cases are rather similar, the robustness over mass and center of gravity changes of the proposed disturbance observer and the load alleviation control algorithm is confirmed.

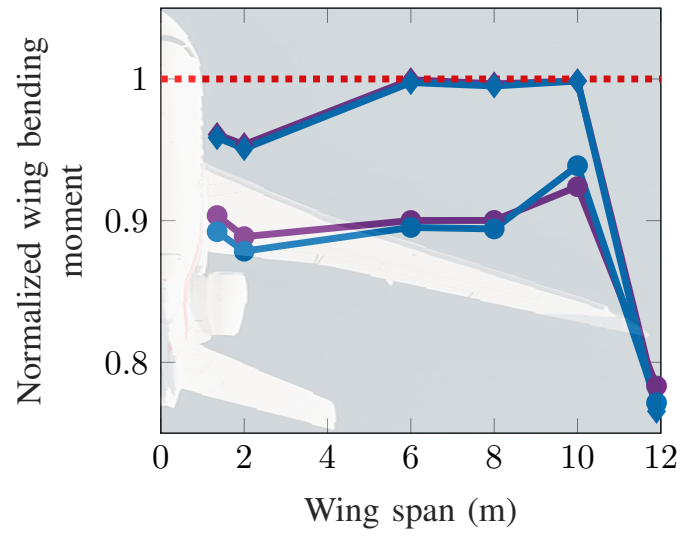

Fig. 4. Load analysis results for the design ( $(-)$ and verification ( $(-)$ case without $(\diamond, \diamond)$ and with $(0, \bigcirc)$ load alleviation controller.

\section{CONCLUSION}

In this paper an approach to design linear disturbance estimators of minimal order using the nullspace based design technique as well as advanced model approximation techniques has been presented. The proposed method has been successfully applied to a generic business jet aircraft model. Based on the estimated disturbance a control strategy has been employed to reduce the maximum wing bending moments encountered due to wind gusts.

\section{ACKNOWLEDGMENT}

This work has been funded within the Joint Technology Initiative JTI Clean Sky 2, AIRFRAME Integrated Technology Demonstrator platform "AIRFRAME ITD" (contract $\mathrm{N}^{\circ}$ CSJU-CS2-GAM-AIR-2014-15-01 Annex 1, Issue B04, 2015) being part of the Horizon 2020 research and Innovation framework program of the European Commission. 


\section{REFERENCES}

[1] G. F. Franklin, J. D. Powell, and A. Emami-Naeini. Feedback Control of Dynamic Systems. Addison-Wesley Longman Publishing, 4th edition, 2002.

[2] E. Schrijver and J. van Dijk. Disturbance observers for rigid mechanical systems: Equivalence, stability, and design. Journal of Dynamic Systems, Measurement, and Control, 124(4):539-548, 2002.

[3] Y. Hori and K. Shimura. Position/force control of multi-axis robot manipulator based on the TDOF robust servo controller for each joint. In Proc. of American Control Conference, Chicago, USA, 1992.

[4] G. H. Hostetter and J. Meditch. On the generalization of observers to systems with unmeasurable, unknown inputs. Automatica, 9:721-724, 1973.

[5] G. Basile and G. Marro. On the observability of linear, time-invariant systems with unknown inputs. Journal of Optimization theory and applications, 2(6):410-415, 1969.

[6] V. Gourishangkar, P. Kudva, and K. Ramar. Reduced-order observers for multivariable systems with inaccessible disturbance inputs. International Journal of Control, 25:311-319, 1977.

[7] C. Wang and M. Tomizuka. Design of Robustly Stable Disturbance Observers Based on Closed Loop Consideration Using H Optimization and its Applications to Motion Control Systems. In Proc. of Ameriacan Control Conference, Boston, USA, 2004.

[8] E. Schrijvert, J. van Dijkt, and H. Nijmeijert. Equivalence of Disturbance Observer Structures for Linear Systems. In Proc. of Conference on Decision and Control, Sydney, Australia, 2000.

[9] H. L. Jones. Failure detection in linear systems. $\mathrm{PhD}$ thesis, Massachusetts Institute of Technology, 1971.

[10] M. A. Massoumnia. A geometric approach to failure detection and identification in linear Systems. $\mathrm{PhD}$ thesis, Massachusetts Institute of Technology, 1986.

[11] J. Chen and R. J. Patton. Robust Model-Based Fault Diagnosis for Dynamic Systems. Kluwer Academic Publishers, 1999.

[12] E. Y. Chow and A. S. Willsky. Analytical redundancy and the design of robust failure detection systems. IEEE Trans. Automat. Control, 29:603-614, 1984

[13] X.-C. Lou, A. S. Willsky, and G. C. Verghese. Optimally robust redundancy relations for failure detection in uncertain systems. Automatica, 22:333-344, 1986.

[14] E. Frisk and M. Nyberg. A minimal polynomial basis solution to residual generation for fault diagnosis in linear systems. Automatica, 37:1417-1424, 2001.

[15] A. Varga. On designing least order residual generators for fault detection and isolation. In Proc. of 16th International Conference on Control Systems and Computer Science, pages 323-330, Bucharest, Romania, 2007.
[16] A. Varga. The nullspace method a unifying paradigm to fault detection. In Proc. of Conference on Decision and Control, Shanghai, China, 2009.

[17] A. Varga. Solving Fault Diagnosis Problems - Linear Synthesis Techniques. Springer International Publishing, 2017.

[18] D. Ossmann and A. Varga. Detection and identification of loss of efficiency faults of flight actuators. International Journal of Applied Mathematics and Computer Sciences, 25(1):53-63, 2015.

[19] A. Varga and D. Ossmann. LPV-techniques based robust diagnosis of flight actuator faults. Control Engineering Practice, 31:135-147, 2014.

[20] D Ossmann, H.-D. Joos, and P. Goupil. Enhanced Sensor Monitoring to Maintain Optimal Aircraft Handling in Case of Faults. AIAA Journal of Guidance, Control, and Dynamics, 40(12):3127-3137, 2017.

[21] A. Varga. A Fault Detection Toolbox for Matlab. In Proc. of CASCD, pages 3013-3018, Munich, Germany, 2006.

[22] A. C. Antoulas, S. Lefteriu, and A. C. Ionita. Model reduction and approximation theory and algorithms, chapter A tutorial introduction to the Loewner framework for model reduction. SIAM, Philadelphia. P. Benner, A. Cohen, M. Ohlberger and K. Willcox Eds, 2016.

[23] V. Dalmas, G. Robert, C. Poussot-Vassal, I. Pontes Duff, and C. Seren. From infinite dimensional modelling to parametric reduced order approximation: Application to open-channel flow for hydroelectricity. In Proceedings of the 15th European Control Conference, Aalborg, Denmak, July 2016.

[24] C. Meyer, J. Prodigue, G. Broux, O. Cantinaud, and C. Poussot-Vassal. Ground test for vibration control demonstrator. In Proceedings of the 13th International Conference on Motion and Vibration Control, Southampton, United Kingdom, July 2016.

[25] A. C. Antoulas. Approximation of Large-Scale Dynamical Systems. Advanced Design and Control, SIAM, Philadelphia, 2005.

[26] S. Gugercin, A C. Antoulas, and C A. Beattie. $\mathcal{H}_{2}$ Model Reduction for Large Scale Linear Dynamical Systems. SIAM Journal on Matrix Analysis and Applications, 30(2):609-638, June 2008.

[27] A. J. Mayo and A. C. Antoulas. A framework for the solution of the generalized realization problem. Linear Algebra and its Applications, 425(2):634-662, 2007.

[28] P. Vuillemin. Frequency-limited approximation of large-scale LTI dynamical models. Ph.D. thesis, Onera, ISAE, Toulouse University, Toulouse, France, November 2014

[29] P. Vuillemin, C. Poussot-Vassal, and D. Alazard. $\mathcal{H}_{2}$ optimal and frequency limited approximation methods for large-scale LTI dynamical systems. In Proceedings of the 6th IFAC Symposium on Systems Structure and Control, pages 719-724, Grenoble, France, February 2013.

[30] C. Poussot-Vassal and P. Vuillemin. Introduction to MORE: a MOdel REduction Toolbox. In Proceedings of the IEEE Multi-conference on Systems and Control, pages 776-781, Dubrovnik, Croatia, October 2012. 